\title{
Problem and Pathological Gambling in a Sample of Casino Patrons
}

\author{
Timothy W. Fong • Michael D. Campos • Mary-Lynn Brecht • \\ Alice Davis • Adrienne Marco • Viviane Pecanha • Richard J. Rosenthal
}

Published online: 12 June 2010

(C) The Author(s) 2010. This article is published with open access at Springerlink.com

\begin{abstract}
Relatively few studies have examined gambling problems among individuals in a casino setting. The current study sought to examine the prevalence of gambling problems among a sample of casino patrons and examine alcohol and tobacco use, health status, and quality of life by gambling problem status. To these ends, 176 casino patrons were recruited by going to a Southern California casino and requesting that they complete an anonymous survey. Results indicated the following lifetime rates for at-risk, problem, and pathological gambling: 29.2, 10.7, and 29.8\%. Differences were found with regards to gambling behavior, and results indicated higher rates of smoking among individuals with gambling problems, but not higher rates of alcohol use. Self-rated quality of life was lower among pathological gamblers relative to non-problem gamblers, but did not differ from atrisk or problem gamblers. Although subject to some limitations, our data support the notion of higher frequency of gambling problems among casino patrons and may suggest the need for increased interventions for gambling problems on-site at casinos.
\end{abstract}

Keywords Pathological gambling prevalence $\cdot$ Casino patrons $\cdot$ Gambling behavior

\section{Problem and Pathological Gambling in a Sample of Casino Patrons}

Whereas there has been significant growth in the amount of prevalence research on gambling problems in North America (Volberg 2004), to our knowledge, few studies have examined the prevalence of problem and pathological gambling among patrons at gambling venues. Further, the most recent study in the US using a gambling venue sample was

T. W. Fong $(\bowtie) \cdot$ M. D. Campos - A. Davis - A. Marco - V. Pecanha - R. J. Rosenthal UCLA Gambling Studies Program, David Geffen School of Medicine, Los Angeles, USA e-mail: Tfong@mednet.ucla.edu

M.-L. Brecht

UCLA Integrated Substance Abuse Programs, David Geffen School of Medicine, Los Angeles, USA

T. W. Fong - M. D. Campos - M.-L. Brecht - A. Davis - A. Marco - V. Pecanha - R. J. Rosenthal Semel Institute for Neuroscience and Human Behavior at UCLA, 760 Westwood Plaza, Suite C8-891, Los Angeles, CA 90095, USA 
reported in the late 1990s (Gerstein et al. 1999) and since that time gambling venues and opportunities to gamble have become increasingly prevalent throughout the US.

Four studies in the literature (Fisher 2000; Gerstein et al. 1999; Oliveira and Silva 2000, 2001), all published nearly 10 years ago, have examined the prevalence of pathological gambling among gambling venue patrons in three countries (e.g., the UK, the US, and Brazil). In summary, these studies indicate that: (a) although more stringent methodology yields lower estimates, the percentage of problem/pathological gambers among casino patrons [2.2\% in the UK (Fisher 2000); $7.9 \%$ in the US (Gerstein et al. 1999); and 43.5\% in Brazil (Oliveira and Silva 2000)] is elevated in these samples relative to the estimates for pathological gambling among the North American general public (0.42-4.0\%) (Gerstein et al. 1999; Petry et al. 2005; Shaffer and Hall 2001; Shaffer et al. 1999; Stucki and RihsMiddel 2007; Welte et al. 2001); (b) many problem/pathological gamblers perceive themselves to have a problem with gambling; (c) despite some recognition of problems with gambling, very few individuals in these samples report having sought treatment for gambling problems, which is consistent with the previous literature on treatment seeking among individuals with gambling problems in the US (Kessler et al. 2008; Slutske 2006); and (d) individuals with pathological gambling are more likely to have substance problems, which is consistent with findings from other studies of gambling and substance abuse (Cunningham-Williams et al. 1998; Grant and Potenza 2005; Petry and Oncken 2002; Potenza et al. 2004; Welte et al. 2001; Welte et al. 2004).

In addition to substance-related disorders, previous research on gambling problems has indicated that suicidality (Ledgerwood et al. 2005), health problems (Larimer et al. 2006; Meyer et al. 2000), and reduced quality of life (Black et al. 2003) are common among individuals with pathological gambling disorder. As part of our survey of casino patrons, we assessed alcohol and tobacco use, health status, and quality of life in order to examine differences along these dimensions by gambling problem status.

Data for the analyses presented in this paper come from a casino located in Southern California. The casino is open $24 \mathrm{~h}$ a day, 7 days a week, 365 days a year. The casino is open to the public, with no membership requirements. Data were collected with the knowledge and authorization of the casino management. The casino offers only un-banked table games with cards.

Data from gambling venues in the US are of interest because documenting high prevalence rates of pathological or problem gambling among casino patrons would argue for the systematic implementation of primary, secondary, or tertiary prevention approaches in gambling settings (e.g., training casino employees to identify problem/pathological gamblers, providing information regarding problem/pathological gambling to patrons, and implementing formal treatment referral procedures for identified problem/pathological gamblers). Alternatively, equal prevalence of problem or pathological gambling among casino patrons relative to individuals in other settings would suggest that settings other than casinos (e.g., bankruptcy courts, substance abuse treatment programs, etc.) may be better locations for prevention and treatment outreach programs for gambling problems.

Based on prior work, our primary hypotheses were that rates of problem and pathological gambling would be higher among casino patrons relative to rates for these disorders found in general population samples; individuals with gambling problems would report more tobacco and alcohol use; and, individuals with gambling problems would report lower ratings for health and quality of life. Our primary focus on the prevalence of gambling problems among casino patrons provides data bearing on the potential need for casino-based problem gambling interventions. Our analyses of substance use, self-reported health, and quality of life provide some context for our data on gambling problem 
frequency by examining commonly co-occurring problems which are important in the treatment of gambling problems.

\section{Methods}

Participants and Procedures

The current analyses are based on a total of 178 individuals surveyed inside a Southern California casino. The mean age for the sample was $37(\mathrm{SD}=12.6)$. Roughly $78 \%$ of participants were male, $40 \%$ were Asian Pacific Islander, 28.5\% were Latino/a, and 31.5\% were White. Over $80 \%$ had at least some college education, and $38 \%$ were married. Seventy percent were employed and nearly $45 \%$ had an income range between 25 and 50 thousand per year. Table 1 presents sample demographics and background characteristics.

Prior to data collection, all instruments and procedures were approved by the UCLA General Campus Institutional Review Board. In order to obtain study participants, research

Table $1 M(\mathrm{SD})$ or $N(\%)$ for sample demographics and background characteristics by problem gambling status

\begin{tabular}{|c|c|c|c|c|}
\hline & NPG & $\mathrm{AR}$ & PrG & $\mathrm{PaG}$ \\
\hline$N(\%)$ & $54(30.3)$ & $52(29.2)$ & 19 (10.7) & $53(29.8)$ \\
\hline Age & $36.6(13.3)$ & $36.7(13.5)$ & $41.8(14.9)$ & $39.3(13.3)$ \\
\hline \multicolumn{5}{|l|}{ Gender** } \\
\hline Male & $33(61.1)$ & $43(82.7)$ & $17(89.5)$ & $47(88.7)$ \\
\hline Female & $21(38.9)$ & $9(17.3)$ & $2(10.5)$ & $6(11.3)$ \\
\hline \multicolumn{5}{|l|}{ Ethnicity* } \\
\hline API & $20(37.0)$ & $15(28.8)$ & $9(47.4)$ & $29(54.7)$ \\
\hline Latino/a & $17(31.5)$ & $14(26.9)$ & $8(42.1)$ & $11(20.8)$ \\
\hline White & $17(31.5)$ & $23(44.2)$ & $2(10.5)$ & $13(24.5)$ \\
\hline \multicolumn{5}{|l|}{ Education } \\
\hline H.S. or less & $9(16.7)$ & $9(17.3)$ & $0(0.0)$ & $9(17.0)$ \\
\hline Some College & $16(29.6)$ & $24(46.2)$ & $10(52.6)$ & $23(43.4)$ \\
\hline College Grad & $29(53.7)$ & $19(36.5)$ & $9(47.4)$ & $21(39.6)$ \\
\hline \multicolumn{5}{|l|}{ Marital status } \\
\hline Married & 23 (42.6) & $20(38.5)$ & $10(52.6)$ & $17(32.1)$ \\
\hline Unmarried & $31(57.4)$ & $32(61.5)$ & $9(47.4)$ & $36(67.9)$ \\
\hline Employed full time & $42(77.8)$ & $34(65.4)$ & $13(68.4)$ & $37(69.8)$ \\
\hline \multicolumn{5}{|c|}{ Income range (in thousands) } \\
\hline $0-\$ 25$ & $10(19.2)$ & $12(23.1)$ & $3(15.8)$ & $12(23.5)$ \\
\hline$\$ 25-50$ & $25(48.1)$ & $23(44.2)$ & $10(52.6)$ & $20(39.2)$ \\
\hline$\$ 50-100$ & $12(23.1)$ & $11(21.2)$ & $5(26.3)$ & $14(27.5)$ \\
\hline$>\$ 100$ & $5(9.6)$ & $6(11.5)$ & $1(5.3)$ & $5(9.8)$ \\
\hline
\end{tabular}

Groupings for table based on NODS score

Ns differ due to missing data

$N P G$ non-problem gamblers, $A R$ at-risk gamblers, $\operatorname{Pr} G$ problem gamblers, $P a G$ pathological gamblers

$* p<0.05 ; * * p<0.01$ 
staff went to a casino in Southern California and set up a table in the casino with a sign reading "gamblers wanted to fill out a survey". Data were collected in eight-hour blocks spanning all hours of the day across three business days (Monday, Tuesday, and Wednesday). After providing informed consent, participants completed a study questionnaire. Although basic demographics were collected, no identifying information was included on the questionnaire in order to maintain anonymity. Participants were given a five dollar gift card for a coffee house in compensation for their time. Thus our sample is one of convenience, rather than one systematically selected.

Measures

\section{UCLA Gambling Survey}

A 19-item self-report survey was administered that included questions on demographics, country of origin, gambling related behaviors (e.g., age first gambled, typical frequency of gambling, reasons for gambling, days gambled in the last 30, money spent on gambling in the last 30 days), family history of gambling problems, cigarettes smoked per day, drinks per week, health, and quality of life. Questionnaire items were chosen to balance comprehensiveness with the amount of time necessary for instrument completion. Items were analyzed individually, with no composite scores generated from these data.

\section{South Oaks Gambling Screen (SOGS)}

The SOGS (Lesieur and Blume 1987) consists of 20 scored items and four un-scored items assessing gambling-related behavior. In our study, we used only the items assessing types of gambling (e.g., cards, betting on animal races, slot machines, etc.) and one item assessing whether or not respondents felt that they had ever had a gambling problem. Items assessing types of gambling could be endorsed as either 'not at all' (scored 0), 'less than once a week' (scored 1), or 'once a week or more' (scored 2). The gambling problem item was assessed as 'Yes' or 'No'.

\section{NORC DSM-IV Screen for Gambling Problems (NODS)}

Questions from the 17-Item NORC DSM-IV Screen for Gambling Problems (Gerstein et al. 1999), for which good psychometric properties have been established, were adapted for self-report administration, resulting in a 10-item, yes-no response format, screening questionnaire for problem and pathological gambling. The 10-items correspond with DSMIV criteria for pathological gambling. Internal consistency for our adapted version of the NODS was excellent for this sample (Cronbach's alpha $=0.90$ ). Based on the work of Gerstein et al. (1999), the following taxonomy was used to categorize respondents: $0=$ non-problem gamblers; 1 or $2=$ at-risk gamblers; 3 or $4=$ problem gamblers; and individuals scoring 5 or more were considered pathological gamblers.

\section{Reasons for Gambling}

An open-ended question asked respondents to write in their reason for gambling. These answers were manually reviewed and categorized by the second author (MDC), then reviewed by the first author (TWF). Results of this categorization indicated that the majority 
of responses (126 of 141 responses) could be categorized as either gambling to make money or gambling for fun/pleasure. A single, dichotomous variable was created using these 126 responses. A value of 1 was coded as 'gambling for money' and a value of 2 was coded for 'gambling for fun/pleasure'. Other responses were not coded due to low frequency.

\section{Alcohol Use}

One item asked respondents 'how many drinks they have per week'. Possible responses were 'none at all', ' $1-5$ ', '6-10', '11-15', and 'more than 15 '.

\section{Tobacco Use}

One item asked respondents 'how much they smoked per day'. Possible responses were 'don't smoke', 'less than 10 cigarettes', '10 cigarettes', or 'more than 20 cigarettes'. Due to a typo, the third category was listed on the instrument as '10 cigarettes'.

\section{Health Status}

One item, phrased 'How would you rate your health?', was used to operationalize health status. This item was scored on a 5-point Likert-type scale ranging from 1 (poor) to 5 (excellent).

Statistical Analyses

\section{Missing Data}

Missing data were dealt with in two ways. First, in the case where an individual had missing items for the NODS, we multiplied the total score for all present items by the following formula: $1+$ (items endorsed positive/number of items endorsed). In cases where the entire scale was missing $(n=2)$, we dropped those cases from analysis.

\section{Analyses}

We first obtained the unweighted percentage of individuals in our sample who scored in the non-problem, at-risk, problem, and pathological gambling range. In order to test for differences in demographics, background characteristics, tobacco use, alcohol use, self-reported health, and quality of life between non-problem, at-risk, problem, and pathological gamblers, we conducted $\chi^{2}$ analyses for categorical variables, Kruskal-Wallis tests for ordinal variables, and $t$ tests or one-way ANOVAs with post-hoc Tukey tests for continuous variables.

\section{Results}

Prevalence of Gambling Problems Among Casino Patrons

Using the NODS as our measure of gambling problem severity, we found that $54(30.3 \%)$ of respondents were non-problem gamblers, 52 (29.2\%) were at-risk gamblers, $19(10.7 \%)$ were problem gamblers, and $53(29.8 \%)$ were pathological gamblers. Thus, just over $40 \%$ 
of our sample of casino patrons reported experiencing at least 3 serious gambling-related symptoms in their lifetime.

Analyses of demographic and background characteristics indicated that at-risk, problem, and pathological gambling were more frequent among males $\left(\chi_{[3]}^{2}=14.9, p<0.01\right)$ and that pathological gambling was more frequent among individuals of Asian Pacific Islander descent $\left(\chi_{[6]}^{2}=13.5, p<0.05\right)$. Education, marital status, employment status, and income were unrelated to the frequency of problem or pathological gambling in this sample (see Table 1). The groups did not differ in age.

\section{Gambling Variables}

Pathological gamblers were more likely to have had a family member with a history of problem gambling $\left(\chi_{[3]}^{2}=20.1, p<0.01\right)$, to have gambled 10 or more days in the previous month $\left(\chi_{[3]}^{2}=17.0, p<0.01\right)$, to have gambled more than $\$ 400.00$ in the previous 30 days $\left(\chi_{[3]}^{2}=33.7, p<0.01\right)$, and were more likely to report that they gambled to make money rather than for fun or pleasure $\left(\chi_{[3]}^{2}=25.3, p<0.01\right)$. Although problem and pathological gamblers were younger at age of first gambling experience, the age difference was not significant. Fully $73.6 \%(n=39)$ of those classified as pathological gamblers reported that they have (or have ever had) a gambling problem $\left(\chi_{[3]}^{2}=77.6, p<0.01\right.$ ); whereas $15.4 \%(n=8)$ and $26.3 \%(n=5)$ of at-risk or problem gamblers reported that they have (or have ever had) a gambling problem. These data are presented in Table 2. With regards to specific gambling activities, results of Kruskal-Wallis tests indicated that individuals with pathological gambling were more likely to report frequently playing cards for money $(p<0.01)$, betting on animals $(p<0.05)$, sports betting $(p<0.01)$, going to a casino $(p<0.01)$, and playing lotto/numbers $(p<0.01)$.

\section{Alcohol and Tobacco Use}

Results for the tobacco use and alcohol use variable analyses are presented in Table 3. Although there was no difference with regards to level of alcohol use between groups, pathological gamblers reported smoking more cigarettes per day relative to other groups as tested using a Kruskal-Wallis test $(p=0.02)$. Moreover, self-identified smokers had significantly higher mean NODS scores than those who reported not smoking $[M=3.9$, $(\mathrm{SD}=3.5)$ vs. $\left.M=2.5,(\mathrm{SD}=3.0) ; t_{[77.7]}=-2.5, p<0.05\right]$.

\section{Self-Rated Health and Quality of Life}

Self-rated health and quality of life data are presented in Table 3. There was a trend for lower self-rated health among pathological gamblers $\left(F_{[2,175]}=3.9, p<0.06\right)$ relative to non-problem gamblers. Self-rated quality of life was lower among pathological gamblers relative to non-problem gamblers $\left(F_{[3,174]}=4.1, p<0.01\right)$, but did not differ between atrisk, problem, and pathological gamblers.

\section{Discussion}

A number of large scale epidemiological studies have been conducted internationally and in the US to determine the prevalence of problem/pathological gambling in the general 
Table 2 Mean (SD) or $N(\%)$ for gambling-related variables by problem gambling status

\begin{tabular}{lcccc}
\hline & NPG & AR & PrG & PaG \\
\hline NODS score & $0.0(0.0)$ & $1.3(0.5)$ & $3.4(0.5)$ & $7.3(1.6)$ \\
Age first gambled & $22.7(9.5)$ & $20.2(8.4)$ & $18.9(9.8)$ & $18.9(8.3)$ \\
Family Hx of PG** & $16(29.6)$ & $17(32.7)$ & $10(52.6)$ & $36(67.9)$ \\
Days gambled (Last month)** & & & & \\
0 days & $26(49.1)$ & $12(23.5)$ & $3(16.7)$ & $0(0.0)$ \\
$1-10$ days & $23(43.4)$ & $30(58.8)$ & $11(61.1)$ & $33(61.1)$ \\
$10-20$ days & $3(5.7)$ & $4(7.8)$ & $2(11.1)$ & $15(27.8)$ \\
20-30 days & $1(1.9)$ & $5(9.8)$ & $2(11.1)$ & $6(11.1)$ \\
Gambled $>\$ 400$ (Last month)** & & & \\
$0-100$ & $36(70.6)$ & $22(43.1)$ & $5(27.8)$ & $5(9.3)$ \\
$100-200$ & $6(11.8)$ & $12(23.5)$ & $5(27.8)$ & $6(11.1)$ \\
200-300 & $3(5.9)$ & $7(13.7)$ & $2(11.1)$ & $5(9.3)$ \\
$300-400$ & $1(2.0)$ & $2(3.9)$ & $0(0.0)$ & $8(14.8)$ \\
$>400$ & $5(9.8)$ & $8(15.7)$ & $6(3.4)$ & $30(55.6)$ \\
Reason for gambling** & & & & $21(61.8)$ \\
To make money & $2(5.7)$ & $14(31.1)$ & $3(25.0)$ & $13(38.2)$ \\
For fun/pleasure & $33(94.3)$ & $31(68.9)$ & $9(75.0)$ & $14(26.4)$ \\
Ever had a gambling problem** & & & $14(73.7)$ & $39(73.6)$ \\
No & $54(100)$ & $44(84.6)$ & $5(26.3)$ & \\
Yes & $0(0)$ & $8(15.4)$ & & \\
\hline
\end{tabular}

Groupings for table based on NODS score

Ns differ due to missing data

Mean with different superscripts significantly different by post hoc Tukey test

$N P G$ non-problem gamblers, $A R$ at-risk gamblers, $\operatorname{Pr} G$ problem gamblers, $P a G$ pathological gamblers

** $p<0.01$

population; however, less work has examined the prevalence of pathological gambling among gambling venue patrons. The current study, using data from a sample of individuals surveyed in a casino setting, sought to determine the frequency of gambling problems in a sample of gambling venue patrons. Further, the current study sought to examine alcohol and tobacco use, health status, and quality of life differences by gambling problem status in this sample.

Based on prior work, our primary hypotheses were as follows: (a) rates of problem and pathological gambling would be higher among casino patrons relative to rates for these disorders found in general population samples; (b) individuals with gambling problems would report higher quantities of tobacco and alcohol use; (c) individuals with gambling problems would report lower ratings for health and quality of life.

Prevalence of Gambling Problems

The results of our analyses supported our first hypothesis related to increased rates of gambling problems among casino patrons relative to the general public. Based on the NODS score classification described above, $30.3 \%$ of respondents were non-problem gamblers, $10.7 \%$ were problem gamblers, $29.2 \%$ were at-risk gamblers, and $29.8 \%$ were 
Table 3 Mean (SD) or $N(\%)$ health-related variables by problem gambling status

\begin{tabular}{lcccc}
\hline & NPG & AR & PrG & PaG \\
\hline $\begin{array}{l}\text { Smoke cigarettes* } \\
\text { Do not smoke }\end{array}$ & $43(81.1)$ & $38(73.1)$ & $15(83.3)$ & $31(57.4)$ \\
$<10$ cigarettes/day & $7(13.0)$ & $7(13.2)$ & $3(5.8)$ & $1(5.6)$ \\
10 cigarettes/day & $0(0.0)$ & $7(13.5)$ & $0(0.0)$ & $6(11.1)$ \\
$>20$ cigarettes/day & $3(5.7)$ & $4(7.7)$ & $2(11.1)$ & $10(18.5)$ \\
Drink alcohol & & & & $7(38.9)$ \\
None at all & $27(50.0)$ & $27(51.9)$ & $8(44.4)$ & $28(51.9)$ \\
1-5 Drinks & $23(42.6)$ & $19(36.5)$ & $2(11.1)$ & $6(11.1)$ \\
6-10 Drinks & $1(1.9)$ & $2(3.8)$ & $0(0.0)$ & $0(0.0)$ \\
11-15 Drinks & $2(3.7)$ & $3(5.8)$ & $1(5.6)$ & $1(1.9)$ \\
15+ Drinks & $1(1.9)$ & $1(1.9)$ & $3.1(1.0)$ & $3.1(1.2)$ \\
Self-rated health & $\dagger$ & $3.3(0.8)$ & $3.8(0.8)^{\mathrm{a}, \mathrm{b}}$ & $3.5(1.1)^{\mathrm{b}}$ \\
Self-rated quality of life** & $4.1(0.9)^{\mathrm{a}}$ & $3.8(0.7)^{\mathrm{a}, \mathrm{b}}$ & & \\
\hline
\end{tabular}

Groupings for table based on NODS score

Ns differ due to missing data

Means with different superscripts significantly different by post-hoc Tukey test

$N P G$ non-problem gamblers, $A R$ at-risk gamblers, $\operatorname{Pr} G$ problem gamblers, $\operatorname{Pa} G$ probable pathological gamblers

${ }^{\dagger} p<0.10 ; * p<0.05 ; * * p<0.01$

pathological gamblers. The NODS questions assessed lifetime gambling-related problems, therefore, these categories reflect lifetime rates of gambling disorders and not necessarily frequency of current gambling disorders.

The prevalence rates obtained in our sample were higher than those obtained in a nationwide casino sample (Gerstein et al. 1999) and in a study of casino patrons in the UK (Fisher 2000). Gerstein and associates found that nationwide, $17.9 \%$ of casino patrons were at-risk gamblers, $5.3 \%$ were problem gamblers, and $7.9 \%$ were pathological gamblers. Fisher (2000) and Gerstein et al. (1999) present weighted prevalence estimates that controlled for the likelihood of being sampled. We did not weight our prevalence estimates because we had no measure of the frequency of casino attendance. Had we weighted our data we may have obtained lower rates of pathological gambling than those we observed using un-weighted data.

Conversely, the rate of pathological gamblers found in the current study was lower than that obtained in a study of problem/pathological gambling done in Brazil (43.5\%) (Oliveira and Silva 2000). Oliveira and Silva $(2000,2001)$ used the SOGS scores to classify pathological gamblers and found a higher rate of probable pathological gamblers than we found in our data. In the current study, we employed the NODS, which has been shown to more strictly define gambling problems (Hodgins 2004); further, the SOGS has been shown to have good agreement with DSM-IV criteria for pathological gambling, but may overestimate gambling problems in non-clinical, general population samples (Stinchfield 2002).

The obtained high rate of pathological gambling in our study may be due to the fact that we had a primarily male sample $(78.7 \%$ male), and that our sample was primarily nonCaucasian (69.1\%). Male gender and non-Caucasian ethnicity have been associated with higher rates of problem gambling (Fisher 2000; Gerstein et al. 1999; Volberg 1996). 
Although in our sample we found that problem gamblers were more likely to be male, just under $16 \%$ of women surveyed in this study were pathological gamblers.

\section{Gambling Behaviors}

Relative to non-problem gamblers, a greater number of pathological gamblers showed heavy current gambling involvement as indicated by gambling 10 or more days in the last 30 and gambling greater than $\$ 400.00$ in the last month. The majority of pathological gamblers $(61.8 \%)$ reported that they gambled to make money.

In the current sample, pathological gamblers were more likely to report 'action' oriented game play (e.g., cards, betting on animals, betting on sports), with the exception that they also reported increased frequency of lottery play. This may be due to the nature of the casino from which data were collected. The casino offers only card games, therefore, it likely attracts those individuals with a primary interest in more action oriented games. Our sample may have a bias towards action oriented gamblers for this reason.

\section{Alcohol and Tobacco Use}

Our hypotheses regarding alcohol and tobacco use were partially supported. Somewhat consistent with other studies, we found that pathological gamblers smoked more cigarettes per day than non-pathological gamblers (Cunningham-Williams et al. 1998; Grant and Potenza 2005; Petry and Oncken 2002; Potenza et al. 2004). We also found that smokers had higher NODS scores relative to non-smokers, which is consistent with the report of more severe gambling pathology among pathological gamblers who smoke (Petry and Oncken 2002). Unlike past work, we did not find a relationship between alcohol use and pathological gambling (Cunningham-Williams et al. 1998; Welte et al. 2001; Welte et al. 2004). The failure to find an association between alcohol use and pathological gambling may be a result of our alcohol use measurement method. Had we employed alcohol abuse or dependence diagnoses, we may have detected a relationship between pathological gambling and alcohol abuse/dependence.

\section{Health and Quality of Life}

We hypothesized that individuals with gambling problems would report worse health than non-problem gambling casino patrons and that individuals with gambling problems would report reduced quality of life relative to non-problem gambling casino patrons. In the case of self-reported health, our hypothesis was not supported. Although pathological gamblers reported lower self-rated health than non-pathological gamblers, the difference was not statistically significant beyond the trend level. But, for self-reported quality of life, our hypothesis was supported. Pathological gamblers reported lower quality of life than nonpathological gamblers. Mean self-reported quality of life scores for the at-risk and problem gambling groups were higher than those reported by the pathological gambling group, but lower than those reported by the non-problem gambling group.

\section{Problem Awareness}

Evidence from our data suggest that individuals with gambling problems were aware of family histories of gambling problems and may have insight into their own gambling 
problems. Significantly more individuals with gambling problems reported that someone in their family had or has a gambling problem. Approximately $84 \%$ of individuals who reported 3 or more gambling-related problems as assessed by the NODS indicated that they felt that they either had or currently have a gambling problem.

Despite the fact that a high percentage of individuals who reported 3 or more gamblingrelated problems also indicated that they felt they may have a gambling problem, it is unlikely that these individuals have sought or will seek treatment. In the US lifetime treatment seeking among individuals with pathological gambling (PG) disorder is low (Kessler et al. 2008; Slutske 2006) as compared with psychiatric disorders such as substance-related disorders and major depression (Kessler et al. 1998). Slutske (2006) compared rates of treatment seeking among individuals with PG surveyed as part of two national studies: the Gambling Impact and Behavior Study (GIBS) and the National Epidemiologic Survey on Alcohol and Related Conditions (NESARC). In the GIBS data only $7.1 \%$ of the lifetime pathological gamblers reported seeking professional treatment or participation in self-help groups. In the NESARC data only $9.9 \%$ of the lifetime pathological gamblers sought professional treatment or had attended at least one Gamblers Anonymous meeting. Kessler et al. (2008), in an analysis of data from the National Comorbidity Survey Replication (NCS-R), found that of study participants meeting lifetime criteria for $\mathrm{PG}$, none reported seeking treatment specifically for gambling problems; however, $49 \%$ of those with a lifetime diagnosis of PG reported treatment for substance disorders or emotional problems at any point in their life. Comparatively, for addictive disorders and major depression, roughly 50 and $70 \%$, respectively, make treatment contact with a physician, mental health professional, or other professional over their lifetime (Kessler et al. 1998).

Among reasons such as embarrassment regarding gambling behavior, denial of a gambling problem, social stigma, and concerns about the effectiveness of treatment, the simple lack of available services has been cited as a barrier to PG treatment utilization (Rockloff and Schofield 2004).

In order to increase awareness of gambling treatment availability, effectiveness, and utilization, casino-based interventions for gambling problems may be necessary. Such interventions could include posting information about available treatment services, formalized screening, identification, and referral procedures for individuals with gambling problems in casino settings, and/or gambling problem screening kiosks at key locations within a casino. The incorporation of curricula on the recognition of the signs and symptoms of problem gambling into standard training practices for casino staff, coupled with a formal procedure to refer interested individuals to gambling treatment services could also be implemented. Our examination of alcohol and tobacco use, health status, and quality of life suggest that interventions for smoking cessation and improving quality of life are needs identified among casino patrons with gambling problems.

\section{Study Strengths and Limitations}

The current research may be characterized as having a number of strengths. First, the data come from a 'real-world' casino setting rather than in a laboratory setting, or a college population. Second, we employed a three-day round-the-clock sampling method that increased the likelihood that individuals with varying gambling patterns would be sampled. Third, we employed a purpose-built measure, the NODS, in order to assess for pathological gambling. The NODS was designed specifically to operationalize pathological gambling 
criteria for community based studies. Finally, all data were collected anonymously, which may have increased the likelihood that respondents would provide accurate and reliable information regarding gambling and associated behaviors.

Findings from the current study must be considered in light of a number of limitations. First, we present data gathered primarily as a convenience sample from a single Los Angeles County casino. Systematic bias may have been introduced in our sample as a result of specific aspects of the casino from which data were sampled, by lack of selection criteria for inclusion in the study, or participant characteristics which may be related to choosing to participate in research surveys. A second limitation was the fact that our data are entirely self-report and may be subject to recall bias, social desirability bias, and other distortions. Finally, the study was limited by the fact that we did not use DSM-based measures for substance disorders in our survey and used single-item quality of life and health status measures. More refined measurement techniques would have allowed for more detailed analysis of group differences within these domains.

\section{Summary and Conclusions}

In the current study of casino patrons, we found higher rates of pathological gambling relative to some previous work (Fisher 2000; Gerstein et al. 1999), but lower rates than work conducted in Brazilian gambling venues (Oliveira and Silva 2000, 2001). Gambling related variables were significantly different in that pathological gamblers were more likely to report a family history of gambling problems, to report more frequent gambling with larger sums of money, to report engaging in sports betting and skilled gambling, and to report gambling to make money. A high percentage of pathological gamblers acknowledged having a gambling problem. Individuals with gambling problems reported more smoking, but not more drinking. Pathological gamblers reported lower quality of life, which may be due in part to a trend for lower self-reported health.

The larger implications of our findings are that, given the potentially high rate of gambling problems among casino patrons, there is a need for formal prevention and intervention measures in casino settings. More data from casino samples may be helpful for state and county policy makers in that they provide information upon which 'evidencebased' legislation regarding gambling regulations and controls could be based. Legislators may serve the public interest and prevent untoward consequences resulting from gambling problems by supporting the implementation of primary, secondary, and tertiary prevention efforts in casino settings. The current study-as well as the studies mentioned in our review of the literature-have identified a high frequency of gambling-related problems among casino patrons. Such problems are associated with poor health, substance use, reduced quality of life, psychopathology, and family/social problems. Carefully designed prevention and treatment programs that account for the significant comorbidity present among individuals with problem/pathological gambling disorders are needed. These programs, at minimum, should cooperate with gambling venues by providing training in recognition of gambling problems among patrons, providing casinos with information to provide treatment referrals for patrons with gambling problems.

Acknowledgments This work was supported by funding from The Annenberg Foundation and the National Institute on Drug Abuse (Grant \#: K23DA19522-2).

Open Access This article is distributed under the terms of the Creative Commons Attribution Noncommercial License which permits any noncommercial use, distribution, and reproduction in any medium, provided the original author(s) and source are credited. 


\section{References}

Black, D. W., Moyer, T., \& Schlosser, S. (2003). Quality of life and family history in pathological gambling. Journal of Nervous and Mental Disease, 191(2), 124-126.

Cunningham-Williams, R. M., Cottler, L. B., Compton, W. M., 3rd, \& Spitznagel, E. L. (1998). Taking chances: Problem gamblers and mental health disorders-results from the St. Louis epidemiologic catchment area study. American Journal of Public Health, 88(7), 1093-1096.

Fisher, S. (2000). Measuring the prevalence of sector-specific problem gambling: A study of casino patrons. Journal of Gambling Studies, 16(1), 25-51.

Gerstein, D., Murphy, S., Toce, M., Hoffman, J., \& Palmer, A. (1999). Gambling impact and behavior study: Report to the National Gambling Impact Study Commission. Chicago: National Opinion Research Center.

Grant, J. E., \& Potenza, M. N. (2005). Tobacco use and pathological gambling. Annals of Clinical Psychiatry, 17(4), 237-241.

Hodgins, D. C. (2004). Using the NORC DSM screen for gambling problems as an outcome measure for pathological gambling: Psychometric evaluation. Addictive Behaviors, 29(8), 1685-1690.

Kessler, R. C., Hwang, I., Labrie, R., Petukhova, M., Sampson, N. A., Winters, K. C., et al. (2008). DSM-IV pathological gambling in the National Comorbidity Survey Replication. Psychol Med, 38(9), 13511360 .

Kessler, R. C., Olfson, M., \& Berglund, P. A. (1998). Patterns and predictors of treatment contact after first onset of psychiatric disorders. American Journal of Psychiatry, 155(1), 62-69.

Larimer, M. E., Lostutter, T. W., \& Neighbors, C. (2006). Gambling in primary care patients: Why should we care and what can we do about it? General Hospital Psychiatry, 28(2), 89-91.

Ledgerwood, D. M., Steinberg, M. A., Wu, R., \& Potenza, M. N. (2005). Self-reported gambling-related suicidality among gambling helpline callers. Psychology of Addictive Behaviors, 19(2), 175-183.

Lesieur, H. R., \& Blume, S. B. (1987). The South Oaks Gambling Screen (SOGS): A new instrument for the identification of pathological gamblers. American Journal of Psychiatry, 144(9), 1184-1188.

Meyer, G., Hauffa, B. P., Schedlowski, M., Pawlak, C., Stadler, M. A., \& Exton, M. S. (2000). Casino gambling increases heart rate and salivary cortisol in regular gamblers. Biological Psychiatry, 48(9), 948-953.

Oliveira, M. P., \& Silva, M. T. (2000). Pathological and nonpathological gamblers: A survey in gambling settings. Substance Use and Misuse, 35(11), 1573-1583.

Oliveira, M. P., \& Silva, M. T. (2001). A comparison of horse-race, bingo, and video poker gamblers in Brazilian gambling settings. Journal of Gambling Studies, 17(2), 137-149.

Petry, N. M., \& Oncken, C. (2002). Cigarette smoking is associated with increased severity of gambling problems in treatment-seeking gamblers. Addiction, 97(6), 745-753.

Petry, N. M., Stinson, F. S., \& Grant, B. F. (2005). Comorbidity of DSM-IV pathological gambling and other psychiatric disorders: Results from the National Epidemiologic Survey on Alcohol and Related Conditions. Journal of Clinical Psychiatry, 66(5), 564-574.

Potenza, M. N., Steinberg, M. A., McLaughlin, S. D., Wu, R., Rounsaville, B. J., Krishnan-Sarin, S., et al. (2004). Characteristics of tobacco-smoking problem gamblers calling a gambling helpline. Am J Addict, 13(5), 471-493.

Rockloff, M. J., \& Schofield, G. (2004). Factor analysis of barriers to treatment for problem gambling. Journal of Gambling Studies, 20(2), 121-126.

Shaffer, H. J., \& Hall, M. N. (2001). Updating and refining prevalence estimates of disordered gambling behaviour in the United States and Canada. Canadian Journal of Public Health, 92(3), 168-172.

Shaffer, H. J., Hall, M. N., \& Vander Bilt, J. (1999). Estimating the prevalence of disordered gambling behavior in the United States and Canada: A research synthesis. American Journal of Public Health, 89(9), 1369-1376.

Slutske, W. S. (2006). Natural recovery and treatment-seeking in pathological gambling: Results of two U.S. national surveys. American Journal of Psychiatry, 163(2), 297-302.

Stinchfield, R. (2002). Reliability, validity, and classification accuracy of the South Oaks Gambling Screen (SOGS). Addictive Behaviors, 27(1), 1-19.

Stucki, S., \& Rihs-Middel, M. (2007). Prevalence of adult problem and pathological gambling between 2000 and 2005: An update. Journal of Gambling Studies, 23(3), 245-257.

Volberg, R. A. (1996). Prevalence studies of problem gambling in the United States. Journal of Gambling Studies, 12(2), 111-128.

Volberg, R. A. (2004). Fifteen years of problem gambling prevalence research: What do we know? Where do we go? The Electronic Journal of Gambling Issues, 10, 1-19. 
Welte, J. W., Barnes, G., Wieczorek, W., Tidwell, M. C., \& Parker, J. (2001). Alcohol and gambling pathology among U.S. adults: Prevalence, demographic patterns and comorbidity. J Stud Alcohol, $62(5), 706-712$.

Welte, J. W., Barnes, G. M., Wieczorek, W. F., Tidwell, M. C., \& Parker, J. C. (2004). Risk factors for pathological gambling. Addictive Behaviors, 29(2), 323-335. 